\title{
Padrões de Uso e Motivos para Envolvimento em Redes Sociais Virtuais na Adolescência
}

\author{
Juliana Szpoganicz Rosado* \\ Universidade Federal do Rio Grande do Sul, Porto Alegre, Brasil \\ Márcia Elisa Jager \\ Universidade Federal de Santa Maria, Santa Maria, Brasil
}

Ana Cristina Garcia Dias

Universidade Federal de Santa Maria, Santa Maria, Brasil

\begin{abstract}
RESUMO
Este trabalho investigou se há diferenças no acesso à internet e uso das redes sociais entre alunos de escolas públicas e privadas e quais os motivos para se conectarem nas mesmas. Um questionário aplicado em quatros escolas buscou compreender a opinião dos jovens sobre o assunto. Os resultados apontaram diferenças no tempo de uso das redes sociais por estudantes de escolas públicas e privadas. Ademais, os alunos indicaram conectar-se nas redes sociais para falar, principalmente, com familiares e amigos já conhecidos. Entende-se que os adolescentes consideram as redes sociais um local de circulação de informações e que é necessária cautela para navegar neste universo cibernético.
\end{abstract}

Palavras-chave: adolescentes; renda familiar; rede social; internet.

\begin{abstract}
Patterns of Use and Reasons for Involvement in Virtual Social Networks in Adolescence This research investigated whether there are differences in internet access and use of social networks among students in public and private schools and what the reasons for connecting same. A questionnaire was applied in four schools in order to understand the views of young people about the subject. Results showed students from public and private schools differ in the amount of time spent in social networks. In addition, students indicated using social networks to chat, specially, with family and friends already known. In this study, it's understood that adolescents considered social networks as a place where can flow information and that caution is require to navigate in the cyber universe.

Keywords: teenagers; family income; social network, internet.
\end{abstract}

$\mathrm{O}$ uso da internet tem se propagado entre indivíduos de diferentes faixas etárias e backgrounds culturais, tornando-se um fenômeno popular de busca pela informação, contato interpessoal e influências sociais imensuráveis. Conforme os dados da Pesquisa Nacional por Amostra de Domicílios (PNAD) realizada pelo Instituto Brasileiro de Geografia e Estatística (IBGE), em 2008, 56 milhões de usuários acessavam a internet no Brasil. A maior parcela das pessoas usuárias da rede eram adolescentes e jovens, entre 10 e 24 anos de idade. Na última pesquisa divulgada pelo IBGE (2010), baseada nos dados da PNAD realizada em 2009, 67,9 milhões de pessoas com 10 ou mais anos de idade declararam ter usado a internet. Esses dados representam um aumento de 12 milhões $(21,5 \%)$, se comparado aos dados apresentados em 2008 sobre o mesmo assunto. No ano de 2009, 71,1\% das pessoas que tinham entre 15 a 17 anos acessavam a rede. Esse crescimento mostra o quanto essa população vem se apropriando da internet e a utilizando com maior frequência.

$\mathrm{O}$ avanço da internet e a rapidez de sua disseminação entre as pessoas trouxeram novas formas de relacionamentos interpessoais, em um novo ambiente de interação virtual, e de circulação de informações, chamado por Levy (1999) de ciberespaço. Para esse autor, o ciberespaço caracteriza-se como um "espaço de comunicação aberto pela interconexão mundial dos computadores e das memórias dos computadores" ( $p$. 92). Esse conceito inclui todas as tecnologias de in-

* Endereço para correspondência: Juliana Szpoganicz Rosado - julianasrosado@gmail.com 
formação e de comunicação digitais mediadas por computador, tais como telefonia clássica e redes hertzianas. O termo "ciberespaço" especifica não apenas a infraestrutura material da comunicação digital, mas também o universo oceânico de informações que ela abriga, assim como as diversas atividades que podem ser desenvolvidas em seu contexto (Lévy, 1999).

A internet, principal ambiente cibernético, oferece diversos atrativos para seus usuários. Em uma pesquisa realizada por Spizzirri, Wagner, Mosmman e Armani (2012), 534 adolescentes indicaram que as principais vantagens no uso desse recurso são: a rapidez, a economia, a possibilidade de conhecer pessoas, de aumentar o número de amigos e que não se precisa ver a pessoa que se encontra no outro lado do computador patra existir uma relação. Nesse estudo, os autores encontraram que $89,6 \%$ dos adolescentes pesquisados têm computador com acesso à internet. Dentre esses que utilizam a rede, " $40,6 \%$ se comunicam por Orkut e MSN (Messenger), sendo que foram classificados na seguinte ordem de preferência 87,64\% MSN, 70,22\% Orkut, 25,4\% e-mail e 3,37\% chats" (p. 331, Spizzirri, Wagner, Mosmman e Armani, 2012).

Outra pesquisa realizada pelo Governo Federal brasileiro indica que os sites de redes sociais são um dos principais serviços utilizados pelos usuários da internet. Em primeiro lugar no ranking de acessos aparece o site de pesquisa e busca Google $(71,6 \%$ dos internautas têm acesso), em segundo lugar as redes sociais e sites de relacionamento $(65,4 \%$ dos internautas se conectam ao Orkut e $64,7 \%$ utilizam o MSN para se comunicarem) (Governo Federal, 2010). Esses recursos, disponíveis no ciberespaço, são capazes de proporcionar aos seus usuário uma facilidade de acesso, velocidade de comunicação, além de possibilitar o compartilhamento de informações sobre o cotidiano (Nicolaci-da-Costa, 1998).

As redes sociais (facebook, twitter, orkut, entre outros) são resultados de transformações tecnológicas e tornaram-se ferramentas importantes de interação interpessoal (Wolton, 2003). Estas redes proporcionam para seus usuários informação e comunicação, além de possibilidades de experimentação de identidades e de estabelecimento de novos relacionamentos com as pessoas (Dias, 2003; Turkle, 1998). Esta nova forma de contato interpessoal, apesar de se configurar em um ambiente virtual, também se faz real, atual e existente, revelando uma maneira diferente de ser, de estar, de se relacionar e de criar laços com o mundo. Assim, atualmente, a rede social é vista como um conjunto de elementos de interação e de relação entre as pessoas (Recuero, 2009).

De fato, as redes sociais - links e sites - nos quais os usuários podem se conhecer e trocar informações sobre si - passam a ser percebidas como ambientes de interação, apreciados principalmente pelo público adolescente. $\mathrm{O}$ uso acelerado das redes sociais por adolescentes pode ser decorrente da própria fase de desenvolvimento humano na qual esses indivíduos se encontram, visto que é neste período que os jovens estão predispostos a investir em relações sociais (Regan \& Steeves, 2010). Allen, Evans, Hare e Mikami (2010) afirmam que é provavelmente nesse período do desenvolvimento que a esfera social da vida de uma pessoa se constrói.

Nesta perspectiva, os adolescentes da contemporaneidade têm se mostrado "diferentes" de gerações passadas. Rodeados pela "vida tecnológica", com computadores, controles remotos, videogames, celulares, tablets e outras tecnologias, os jovens lidam com recursos que até então eram inexistentes em gerações passadas. A partir disto, nos deparamos com uma nova maneira de se viver a adolescência, atravessada constantemente pelas influências destas tecnologias na vida dos jovens (Enstein \& Estefenon, 2006, Ponte, 2010).

Lembramos que a adolescência, delimitada cronologicamente pela Organização Mundial da Saúde como um período do desenvolvimento humano situado entre os 10 e 19 anos de idade (Who, 2012), é considerada como uma fase de descobertas e experimentações. Assim, as interações sociais, estabelecidas no contexto presencial e virtual, assumem um importante papel na vida dos jovens na medida em que oferecem um espaço para experimentação, reflexão e (re)construção de si mesmo e de suas relações interpessoais (Dias, 2003).

As redes sociais virtuais possibilitam o contato com novas pessoas e a ampliação do círculo de relacionamentos, sem que, ao menos, o indivíduo precise sair do lugar no qual está (Lévy, 1999). Neste sentido, elas se tornam atraentes principalmente para os adolescentes que buscam em suas relações sociais a base para consolidação de suas identidades. Através da internet e das redes sociais virtuais é possível criar novas identidades e assumir diferentes papéis (Nobrega, 2010; Turkle, 1998). É durante a adolescência que 
o sujeito experimenta diversos papéis antes de atingir um senso estabelecido de eu (Erikson, 1972). Assim, através das redes, os jovens podem moldar-se e criarse constantemente, até atingir uma identidade satisfatória que esteja de acordo com suas necessidades e crenças (Turkle, 1998).

Nesse sentido, o uso das redes sociais disponíveis na internet é um comportamento comum entre adolescentes. As estatísticas do governo federal (2010) apontam que entre os jovens de 16 e 24 anos, 68,\% deles acessam a internet. Esse dado é congruente com pesquisa realizada pelo Datafolha, publicada em 2013, que revela que $83 \%$ dos jovens de 16 e 17 anos acessam a internet. Estes jovens buscam principalmente se conectar nos sites de relacionamentos, como Orkut e Facebook (Datafolha, 2013). No relatório de pesquisa do Governo Federal sobre os hábitos de informação e formação de opinião da população brasileira observase ainda que quanto maior a renda familiar dos usuários, maior a oportunidade de acesso à internet (Governo Federal, 2010).

Considerando esses aspectos, este estudo pretende investigar se há diferenças no tempo de uso das redes sociais pelos adolescentes de escolas públicas e privadas e quais os motivos pelos quais eles se conectam nas mesmas. O interesse pelo tema justifica-se uma vez que são os jovens os que mais acessam as redes sociais, e também porque há evidências de que a renda familiar tem relação com as oportunidades de acesso à internet. Assim, buscou-se investigar a existência de diferenças no uso de redes sociais por parte de alunos de escolas públicas e privadas, considerando que o tipo de escola é um indicador aproximado de renda familiar. Além disso, foram investigadas possíveis diferenças nos motivos relacionados à utilização das redes sociais nos adolescentes destas escolas.

\section{MÉTODO}

Esta pesquisa baseia-se em um delineamento descritivo quantitativo. Busca tanto descrever as características e os motivos para o uso de redes sociais em adolescentes como comparar essas variáveis, considerando o tipo de escola ou renda familiar dos usuários. Esse delineamento procura conhecer os fenômenos, quantitativamente, a partir da construção de hipóteses sobre as variáveis escolhidas para o estudo. Nesse sentido, busca mensurar e/ou comparar opiniões, atitudes e preferências comportamentais, além de estabe- lecer relações entre variáveis (Richardson et al., 2007).

\section{Participantes}

Participaram 277 estudantes do ensino médio (52,2\% do sexo feminino), com idades entre 13 e 19 anos $(\mathrm{M}=16,2 ; \mathrm{DP}=1,20)$. Os estudantes estavam matriculados em escolas públicas $(44 \%)$ e particulares (46\%) de uma cidade do interior do Estado do Rio Grande do Sul (RS).

\section{Instrumentos e Procedimentos}

Utilizou-se um questionário elaborado exclusivamente para este estudo a partir da análise de literatura sobre redes sociais virtuais e sua influência na vida dos adolescentes. O instrumento constitui-se por questões fechadas sobre o padrão e motivos de uso das redes sociais virtuais e a opinião dos adolescentes a respeito do impacto das redes em diferentes áreas de suas vidas, como relacionamentos interpessoais e atividades de lazer. Para este estudo foram analisadas apenas as questões relacionadas a padrão (frequência) e motivos de uso das redes sociais virtuais.

Escolas públicas e privadas de uma cidade do interior do RS foram contatadas para a realização da pesquisa. Após obtida autorização institucional para a coleta de dados, a aplicação dos questionários foi realizada em sala de aula de forma coletiva. Todos os participantes do estudo assinaram o Termo de Consentimento Livre e Esclarecido, no qual estão descritos os riscos e benefícios de participar do estudo, bem como lhes foi oferecida à garantia de sigilo e confidencialidade das informações prestadas. Não se considerou a necessidade de um responsável assinar o termo, visto que o estudo apresentou um risco ínfimo aos jovens, além de tratar-se de um tema atualmente comentado nas mídias. Além disso, os adolescentes participantes do estudo poderiam compreender os objetivos, justificativa e procedimentos do estudo, estando, assim, em condições de ter autonomia para assegurar sua participação. A pesquisa foi aprovada pelo Comitê de Ética da Universidade Federal de Santa Maria (UFSM), RS, conforme parecer $\mathrm{n}^{\mathrm{o}} 23.081 .020 / 2011-00$.

\section{Análise dos dados}

Utilizou-se o teste de qui-quadrado ou o teste exato de Fisher para verificar as possíveis associações entre o tipo de escola (pública ou privada) e as variáveis 
relacionadas ao padrão e motivo de uso das redes sociais virtuais. Consideraram-se como estatisticamente significativos resultados com $\mathrm{p}<0,05$.

\section{RESULTADOS}

\section{Diferença no uso das redes sociais entre alunos de escolas públicas e privadas}

A fim de investigar possíveis associações entre o padrão de uso das redes sociais e o tipo de escola (pública ou privada) foram realizados testes de quiquadrado. A Tabela 1 apresenta a frequência de uso da internet de acordo com o tipo de escola. Foi verificada uma associação estatisticamente significativa entre estas variáveis $\left(\chi^{2}=21,4 ; \mathrm{gl}=4 ; \mathrm{p}<0,001\right)$.

\begin{tabular}{|c|c|c|}
\hline Frequência de Uso & Escola Pública (\%) & Escola Particular (\%) \\
\hline Não utilizo & 8,0 & 0 \\
\hline Até uma vez por semana & 15,6 & 3,2 \\
\hline De 2 a 3 vezes por semana & 22,1 & 14,8 \\
\hline De 4 a 6 vezes por semana & 17,2 & 15,5 \\
\hline Todos os dias & 44,3 & 56,0 \\
\hline
\end{tabular}

Os dados representados nesta tabela permitem observar que $8 \%$ dos alunos matriculados na escola pública não utilizavam a internet, enquanto que nas escolas particulares não houve estudantes que referissem a não utilização desse recurso. Os dados também apontam que $15,6 \%$ dos alunos de escolas públicas utilizavam a internet até uma vez por semana, enquanto apenas 3,2\% dos estudantes das escolas particulares acessam a rede somente uma vez. Essas percentagens são expressivas e podem sugerir que os fatores socioeconômicos (baixa renda familiar e ausência da possibilidade de acesso à internet em casa) podem estar influenciando na utilização mais frequente ou não da internet e, consequentemente, na utilização das redes sociais. De fato, foi verificada uma associação significativa entre o tipo de escola e a renda familiar $(\chi 2=$ $152,8 ; \mathrm{gl}=3 ; \mathrm{p}<0,001)$. Na escola particular predominou a renda acima de $\mathrm{R} \$ 3.270,00(78,1 \%)$, já na escola pública a renda predominante foi de $\mathrm{R} \$ 545,00$ a R\$1635,00 (58,6\%).

Em relação à possibilidade de acesso à internet em casa (ter um computador para se conectar na rede) na escola particular verificou-se que todos os alunos (100\%) tinham acesso a esse recurso. Na escola públi- ca 71,3\% dos alunos tinham possibilidade de conexão na internet no seu próprio domicílio $(\chi 2=50,9 ; \mathrm{gl}=1$; $\mathrm{p}<0,001)$. Essas percentagens confirmam que a renda pode contribuir para a utilização mais frequente ou não da internet, pois a maior renda pode estar associada a uma maior disponibilidade de recursos materiais (computador) e oportunidades de acesso à internet. Encontrou-se ainda que todos os estudantes das escolas particulares possuíam computador em casa, enquanto que somente $86,1 \%$ dos alunos da escola pública o tinham $(\chi 2=23,0 ; \mathrm{gl}=1 ; \mathrm{p}<0,001)$. Os dados indicam uma clara correlação entre posse de computador e renda: os que possuíam computador tinham uma renda familiar superior à renda dos que não possuíam.

Tratando-se diretamente da utilização das redes sociais, como Facebook, Twitter e Orkut, a Tabela 2 apresenta informações semelhantes à Tabela 1 no que se refere à utilização desses recursos e tipo de escola. Observa-se que os alunos das escolas particulares eram usuários mais frequentes das redes sociais do que os estudantes das escolas públicas $(\chi 2=9,2 ; \mathrm{gl}=$ $3 ; \mathrm{p}=0,027)$. 
Tabela 2

Associação Entre Tipo de Escola e Frequência no Uso das Redes Sociais

\begin{tabular}{lcc}
\hline $\begin{array}{l}\text { Frequência de Uso das } \\
\text { Redes Sociais }\end{array}$ & Escola Pública (\%) & Escola Particular (\%) \\
\hline Não utilizo & 0,8 & 3,3 \\
Até uma vez por semana & 18,9 & 8,5 \\
De 2 a 6 vezes por semana & 34,4 & 31,4 \\
Todos os dias & 45,9 & 56,9 \\
\hline
\end{tabular}

A Tabela 3 apresenta o tempo de acesso (em horas) para o acesso e navegação nas redes sociais. utilizado pelos alunos de escolas públicas e privadas

Tabela 3

Associação Entre Tipo de Escola e Horas Despendidas nas Redes Sociais

\begin{tabular}{lcc}
\hline Horas Conectado nas & Escola Pública (\%) & Escola Particular (\%) \\
Redes Sociais & 0,8 & 3,3 \\
\hline Não utilizo & 55,4 & 66,2 \\
Até 3 horas & 33,9 & 25,8 \\
Entre 3 e 7 horas & 9,9 & 4,6 \\
Mais de 7 horas & & \\
\hline
\end{tabular}

Os dados apresentados nesta tabela mostram que não houve diferenças significativas nas horas despendidas no acesso às redes sociais, pelos alunos de escolas públicas e privadas $(\chi 2=7,3 ; \mathrm{gl}=3 ; \mathrm{p}=0,062)$. A maior parte dos estudantes ficava conectada nas redes até 3 horas por dia. A segunda maior prevalência foi de alunos que indicavam ficar entre 3 e 7 horas por dia nas redes.

A Tabela 4 apresenta as relações entre o tipo de escola e as diferentes redes sociais utilizadas pelos estudantes. O MSN e o Orkut foram as redes sociais mais utilizadas pelos estudantes (mais de $80 \%$ dos participantes indicaram utilizar essas redes sociais). Testes de qui-quadrado indicaram diferenças significativas para uso do Twitter, Facebook e Skype. A maior utilização desses recursos por alunos de escolas particulares provavelmente está relacionado ao tipo de conexão que esses alunos possuíam; a maior renda familiar provavelmente possibilita uma conexão à rede mais rápida e estável, o que por sua vez facilitou eu estes adolescentes utilizassem esses recursos.

Tabela 4

Associação Entre Tipo de Escola e as Ferramentas das Redes Sociais

\begin{tabular}{lccc}
\hline Redes Sociais & Escola Pública (\%) & Escola Particular (\%) & $\boldsymbol{P}$ \\
\hline MSN & 94,3 & 93,5 & 0,806 \\
Orkut & 88,5 & 84,5 & 0,336 \\
Twitter & 47,9 & 60,0 & 0,046 \\
Facebook & 42,1 & 61,9 & 0,001 \\
Skype & 13,2 & 27,1 & 0,005 \\
Blog & 15,8 & 12,3 & 0,394 \\
Formspring & 23,1 & 19,4 & 0,444 \\
Batepapo & 5,8 & 1,9 & 0,089 \\
\hline
\end{tabular}




\section{Motivos que levam os alunos a conectarem-se nas redes sociais}

Para avaliar os motivos que levavam os estudantes a utilizarem as redes sociais, foram oferecidas três respostas possíveis para cada questão: "raramente ou nunca"; "às vezes" e "sempre". A Tabela 5 apresenta os motivos e as frequências de respostas oferecidas em cada questão.

Tabela 5

Associação Entre Tipo de Escola e Motivos Para Utilização das Redes Sociais

\begin{tabular}{lrrrrrrc}
\hline \multirow{2}{*}{ Motivos para utilizar as redes sociais } & \multicolumn{3}{c}{ Escola Pública (\%) } & \multicolumn{3}{c}{ Escola Particular (\%) } \\
\cline { 2 - 8 } & RN & AV & S & RN & AV & S & $P$ \\
\hline Conversar com amigos e familiares & 6,7 & 16,8 & 76,5 & 5,2 & 21,4 & 73,4 & 0,579 \\
Me divertir & 7,5 & 32,5 & 60,0 & 2,0 & 30,7 & 64,1 & 0,070 \\
Baixar músicas e filmes & 8,4 & 35,3 & 56,3 & 3,9 & 24,0 & 65,2 & 0,020 \\
Me sentir melhor quando estou triste ou abatido & 20,3 & 48,3 & 31,4 & 29,4 & 43,1 & 27,5 & 0,235 \\
Poder abstrair das frustrações e correrias do dia-a-dia & 30,3 & 48,7 & 21,0 & 26,8 & 47,7 & 25,5 & 0,648 \\
Poder trocar informações com pessoas do mundo todo & 47,9 & 34,5 & 17,6 & 33,8 & 42,2 & 24,0 & 0,058 \\
Evitar a solidão & 54,6 & 31,1 & 14,3 & 48,4 & 40,5 & 11,1 & 0,261 \\
Conhecer pessoas novas & 30,0 & 58,3 & 11,7 & 41,8 & 52,3 & 5,9 & 0,058 \\
Conseguir promoções e atendimentos mais rápidos e eficazes & 56,3 & 36,1 & 7,6 & 58,8 & 35,9 & 5,2 & 0,720 \\
Poder inventar coisas e mentir & 91,6 & 5,8 & 2,5 & 86,4 & 11,7 & 1,9 & 0,249 \\
\hline
\end{tabular}

Nota: RN - raramente ou nunca; $A V$ - às vezes; $S$ - sempre.

Ao analisarmos os motivos indicados para uso das redes sociais, observamos que, no geral, não houve diferenças significativas entre os alunos de escolas públicas e privadas quanto aos motivos de conexão nas redes sociais. Os motivos presentes na Tabela 5 indicam como os jovens estudantes percebiam o uso das redes sociais na construção de seus relacionamentos interpessoais. Mais da metade dos estudantes afirmaram que às vezes utilizavam a rede para "Conhecer pessoas novas". A rede social também era uma ferramenta muito utilizada para os adolescentes "Conversar com familiares ou amigos".

Esses dados sugerem que os alunos utilizavam as redes sociais para falar com pessoas já conhecidas e para manter relacionamentos já existentes, não descartando que eventualmente poderiam utilizar as redes para se conectar e interagir com pessoas desconhecidas. Contudo, essa não pareceu ser uma prática corriqueira, na medida em que os estudantes descreveram que não costumavam utilizar a rede social para "Poder trocar informações com pessoas de todo o mundo".

Ademais, "evitar a solidão" não foi um motivo frequentemente citado para uso das redes sociais (aproximadamente $50 \%$ dos estudantes de ambas as escolas indicaram nunca usar a rede por esse motivo). Contudo, esse recurso pode ser um motivo que eventualmente ('talvez') pode levar os adolescentes a acessarem as redes sociais. Assim, as redes sociais, por proporcionarem contatos e interações instantâneas, podem servir como uma forma de distração ou para espairecer dos problemas. Essa hipótese pode ser sustentada também pelas altas frequências de concordância com as afirmações: "me sentir melhor quando estou triste ou abatido" (48,3\% dos estudantes da rede pública e $43,1 \%$ das escolas particulares utilizam "às vezes") e "poder abstrair das frustrações do dia-a-dia" (48,7\% dos estudantes da rede pública e $47,7 \%$ das escolas particulares utilizam "às vezes").

"Poder inventar coisas e mentir" não foi uma das principais motivações citadas pelos jovens para conectar nas redes sociais, além disso, mais de $50 \%$ da amostra total das duas escolas, afirmaram que "raramente ou nunca" utilizavam as redes sociais para conseguir promoções de produtos e atendimentos mais rápidos ou eficazes. Estes dados podem retratar a cautela utilizada pelos adolescentes no uso da rede. Além disso, os adolescentes pareceram não conferir grande credibilidade para algo/alguém que não conhecem ou que não interagem presencialmente. 
Por fim, mais de $60 \%$ dos adolescentes dos dois grupos afirmaram que "sempre" utilizavam as redes sociais para se divertir e baixar filmes e músicas. Isso sugere que esse recurso foi efetivamente utilizado para fins recreativos.

\section{DISCUSSÃO DOS RESULTADOS}

A partir dos resultados sobre a frequência em que os alunos utilizavam à internet, percebe-se uma associação entre o tipo de escola e a frequência de uso. Alunos de escolas particulares, com renda familiar superior a $\mathrm{R} \$ 3.270,00$ acessavam mais a internet do que alunos de escolas públicas, com renda familiar de $\mathrm{R} \$ 545,00$ a $\mathrm{R} \$ 1.635,00$. Contudo, percebe-se que um percentual significativo de estudantes, em ambas as escolas e independente da renda familiar, utilizavam a internet todos os dias. Essa informação é similar a encontrada na pesquisa desenvolvida pelo Governo Federal sobre os hábitos de informação e formação de opinião da população brasileira. Esta pesquisa revela que quanto maior a renda familiar dos usuários, maior a oportunidade de acesso à internet (Governo Federal, 2010).

Além disso, os dados confirmam os achados de Clarke-Pearson e O'keeffe (2011). Esses pesquisadores indicam que o uso das redes sociais apresentou um aumento significativo nos últimos cinco anos, tornando-se uma atividade rotineira na vida de crianças e adolescentes. Isto porque a internet vem proporcionando diferentes atrativos para todas as idades, sendo utilizada como meio de comunicação, interação e, até mesmo, aquisição de habilidades técnicas.

Outra pesquisa sobre os acessos e as práticas digitais de adolescentes e jovens com idades entre 15 e 24 anos, realizada em Portugal, também demonstrou, como esse estudo, que existem semelhanças e diferenças nos modos como os jovens de diferentes meios socioeconômicos e culturais utilizam a Internet e as redes sociais. No estudo português, jovens com maior renda familiar têm maior posse de bens tecnológicos e seu comportamento de acesso recebe maior monitoramento parental que adolescentes que possuem renda familiar inferior.

Além disso, o comportamento de busca, a presença de uma visão crítica sobre a informação acessada e o aproveitamento do material digital parecem ser maiores entre os jovens que possuem um capital cultural superior. Contudo, o acesso à rede é semelhante entre os diferentes participantes do estudo no que se refere a busca por comunicação e por entretenimento (Ponte, 2010).

Em relação ao tempo de acesso, a maior parte dos estudantes respondentes desta pesquisa dedicava até três horas de seus dias para a utilização das redes sociais, sem contar o acesso à internet para outras atividades. Spizzirri et al. (2012) encontraram dados similares a estes. Apesar de o estudo desses autores ter investigado tempo de conexão à internet apenas, podemos supor que, como os jovens costumam desenvolver múltiplas atividades durante o período de acesso à internet, os mesmos podem tanto estar conectados as redes sociais exclusivamente, como estar realizando diferentes atividades (por exemplo - consultar um site de busca de informação). Novos estudos podem buscar especificar melhor informações sobre esse tema.

Por outro lado, aproximadamente $35 \%$ dos adolescentes desse estudo afirmaram utilizar as redes sociais entre 3 e 7 horas por dia. Este comportamento também foi verificado em uma pesquisa realizada por pesquisa de Clarke-Pearson \& O'keeffe (2011). No estudo destes autores, $22 \%$ dos adolescentes se conectam as suas redes sociais preferidas mais de 10 vezes ao dia, o que sugere um tempo significativo dispensado por esses jovens no uso da rede.

Como consequência, o maior tempo de uso da internet pode estar associado a maiores riscos para seus usuários. Alguns autores consideram que a internet pode isolar os indivíduos, limitando suas relações sociais e levando a comportamentos dependentes e a um desinvestimento da realidade cotidiana (Leon \& Rotunda, 2000; Turkle, 1998). Neste sentido, a internet estaria colocando em risco a vida privada, pois informações pessoais podem ser obtidas com o uso de má-fé e posteriormente utilizadas para causar danos às pessoas. É o caso, por exemplo, de indivíduos pedófilos ou com outras patologias mentais que podem encontrar suas potenciais vítimas através da rede (Griffits, 1997). Existe ainda a ausência de segurança a respeito de dados confidenciais dos usuários, o que pode gerar formas de controle social. Por exemplo, Griffits (2000) relata que crianças nos Estados Unidos, além de estarem sujeitas à exposição da pornografia, podem ser exploradas por comerciantes através da Internet. Comerciantes utilizam esse meio para obter informações privadas de crianças através de sites direcionados a essa população, com o objetivo de 
traçar perfis individuais e criarem propagandas voltadas especificamente para elas. Além disso, os jovens que utilizam a rede podem estar sujeitos a problemas como cyberbullying e à exposição à pornografia, além de comportamentos de dependência da própria internet (Clarke-Pearson \& O'keeffe, 2011, Pujol, Schmidt, Skolovsky, Karam, \& Spritzer, 2009). Atualmente se fala, inclusive, da Dependência da Internet (DI), que está sendo considerada como um transtorno psiquiátrico do século XXI. Seus sintomas podem ser descritos por preocupação excessiva com o uso da internet; necessidade de aumentar o tempo conectado (online) para ter a mesma satisfação; exibir esforços repetitivos para diminuir o tempo de uso da internet e presença de irritabilidade e/ou depressão na ausência ou impossibilidade de conexão. Além disso, quando o uso da Internet é restringido, o usuário dependente pode apresentar labilidade emocional (internet como forma de regulação emocional); permanecer mais tempo conectado (online) do que o programado; ter o trabalho e relações sociais em risco pelo uso excessivo e mentir aos outros em relação ao tempo de horas online. Cinco ou mais destes critérios respondidos de forma positiva, revelariam Dependência da Internet (Young, 2007).

Entretanto, apesar dos riscos presentes na possibilidade de uso das redes sociais, destaca-se que a utilização deste recurso também pode trazer benefícios aos jovens. A internet e o acesso às redes sociais podem potencializar a comunicação, a busca de informações e o acesso aos serviços online, às atividades de lazer, à compra e venda de produtos. Essas são as principais atividades realizadas pelos adolescentes de Florianópolis, considerados usuários leves da rede, e, portanto, saudáveis (Mello, 2007).

O uso da internet e das redes sociais podem ser vistos como aliados no desenvolvimento dos adolescentes, na medida em que esses ambientes podem funcionar como contextos de criatividade, discussões, formações de opiniões e aprimoramento da escrita (Freitas, 2005). Nesse sentido, o desenvolvimento cognitivo dos adolescentes também pode ser beneficiado com o uso desses recursos, na medida em que são realizadas várias atividades simultaneamente (navegar na rede, bater papo pelo Skype, conversar no MSN, ouvir música), promovendo novas conexões neurais. Essas atividades possibilitam a aquisição de habilidades, como a distribuição da atenção e a agilidade do pensamento (Basmage, 2010; Delaunay, 2008; Ramos \& Silva, 2011).

Em relação ao tipo de redes sociais utilizadas pelos estudantes desta pesquisa, o MSN e o Orkut foram descritas como os recursos preferidos. Este dado também foi encontrado em uma pesquisa realizada com 534 adolescentes de ambos os sexos, de escolas públicas e privadas com idades entre 12 e 17 anos sobre o uso da internet (Spizzirri et al., 2012). Nesse estudo foi encontrado que $40,6 \%$ dos adolescentes se comunicam utilizando esse recurso.

De fato, o uso de Orkut, do MSN e de outros meios de comunicação da internet pelos adolescentes ocorre precocemente, sendo fruto de fascínio por esses recursos. O jovem reconhece tanto a possibilidade de contato rápido, fácil e acessível aos parentes e amigos distantes como o leque de possibilidades de interação sociais propiciadas por esse recurso que permite superar as barreiras do espaço geográfico e do tempo sincrônico (Dias, 2003). Recursos, a exemplo do Orkut, permitem também que os jovens criem uma representação virtual de si, "vistam" e "dispam" as diferentes identidades que desenvolvem nesse contexto. O usuário ainda pode selecionar com que pessoas interagir e o que mostrar para cada um individualmente (Barros $\&$ Neto, 2010).

Além disso, um estudo realizado com estudantes da UFAL (Universidade Federal do Alagoas) indicou que os jovens consideram o Orkut como uma fonte importante de informação e de auxílio na formação acadêmica (Amorin, 2009). Todavia, ressalta-se que se deve avaliar com cuidado as informações que circulam no ambiente virtual, quando elas podem ser utilizadas na vida acadêmica, considerando a cientificidade das mesmas. Prilla, Muza e Campos-Antoniassi (2011) observam que na era da cybercultura, o ambiente e os aparelhos virtuais são amplamente reconhecidos e possibilitam a democratização do conhecimento, sendo considerados como ferramentas de aprendizagem e educação para toda população.

No que se refere ao uso do MSN especificamente, Barros e Neto (2010), em um estudo com seis adolescentes usuários desse programa que avaliou os relacionamentos estabelecidos no MSN, encontraram a descrição de novos processos de subjetivação desencadeados pelo uso desse recurso. Três dimensões explicam esse processo, na concepção dos autores: as experiências tecnológicas de si, o MSN como lugar de 
encontro subjetivo e os processos de subjetivação presentes nesse contexto. $\mathrm{O}$ agenciamento adolescente-MSN se constitui como um laboratório de experimentação subjetiva, que pode produzir tanto intensificações quanto amortecimentos subjetivos, de acordo com a forma como é utilizado e do sentido conferido por seus usuários a esse uso e aos conteúdos que circulam nesse ambiente.

No que se refere aos motivos que levam os estudantes a utilizarem as redes sociais, não foram encontradas diferenças significativas entre os alunos de escolas públicas e privadas. No geral, o objetivo de manter relacionamentos já existentes, tanto com amigos quanto com familiares, teve alta prevalência nos motivos que levam os jovens, de ambas as escolas, a utilizarem as redes sociais. Esses dados são congruentes com outros estudos que apontam que, enquanto alguns adolescentes utilizam as redes sociais para se comunicar com estranhos (Wolak, Mitchell \& Finkelhor, 2003), a maioria deles utiliza para interagir com pessoas já conhecidas (Dias, 2003, Valkenburg \& Peter, 2007).

Valkenburg e Peter (2007) vêm questionando se a internet cria e mantêm relacionamentos de boa qualidade ou se isola as pessoas, diminuindo a qualidade dos relacionamentos sociais e prejudicando o estabelecimento de novas interações. Kraut, Patterson, Lundmark, Kiesler, Mukophadhyay e Scherlis (1998) descrevem que o uso massivo da internet diminuiu a comunicação dos usuários com seus familiares e com membros de seu circulo social presencial, aumentando episódios de depressão e solidão. Bauman (2004), por sua vez, afirma que hoje em dia as pessoas não buscam mais "relacionamentos reais", mas sim "conexões", chamadas de virtuais pelo autor. As relações virtuais seriam baseadas em uma lógica de fluxo e não de qualidade, não havendo construção de intimidade, confiança e compromisso entre as partes. Ele considera que as características desses relacionamentos mediados pelas novas tecnologias são os modelos para os relacionamentos presenciais na atualidade. Hoje em dia haveria uma tendência à fluidez, com a possibilidade das "conexões" interpessoais serem criadas ou jogadas fora como se nunca houvessem existido, com a facilidade de um simples clicar de botão.

Nicolaci-da-Costa (2005) discorda desses autores, para ela os relacionamentos virtuais não são necessariamente superficiais. A autora indica, inclusive, que há diversas pesquisas que concluem exatamente o contrário. Essas pesquisas apontam que essas novas formas de se relacionar via rede podem ser solidárias, profundas e intensas. Desta forma, os relacionamentos virtuais não são necessariamente superficiais, mas se constituem como contextos que criam possibilidades de aprofundar os relacionamentos já existentes e torná-los mais fortes e sustentáveis.

Desse modo, as redes sociais podem ser consideradas espaços para o estabelecimento de contatos verdadeiros, nos quais são estabelecidas conexões com outras pessoas, preferencialmente conhecidas. Em pesquisa realizada em 2007, os autores encontraram que a comunicação nas redes sociais parece manter os aspectos positivos das amizades e dos relacionamentos amorosos estabelecidos presencialmente. De fato, os jovens tendem a utilizar as redes para se conectar com grupos já conhecidos, visando permanecer com seus bons relacionamentos e aprofundá-los (Blais, Craig, Pepler, \& Connolly, 2008).

Dias (2003), em um estudo que investigou as opiniões de adolescentes e jovens sobre internet e salas de bate-papo encontrou que esses contextos são percebidos como "locais" de diversão e de encontro com parceiros de suas relações face a face (ou não) que possuem características demográficas e interesses similares. As conversas estabelecidas no ambiente virtual geralmente versam sobre gostos e interesses, temas gerais e o cotidiano. Assuntos da esfera íntima podem ser abordados nestes contextos, mas são preferencialmente realizados com pessoas conhecidas e de confiança. A comunicação desenvolvida nas salas de bate-papo é percebida de forma similar ao telefone, tendo como vantagem sobre esse meio a possibilidade de interação com várias pessoas a um custo reduzido, na opinião dos jovens.

Observa-se que os dados referentes aos motivos de uso encontrados nesse estudo são similares aos encontrados nos estudos descritos anteriormente (Dias, 2003; Mello, 2007; Spizzirri et al., 2012; Ramos \& Silva, 2011). A internet e as redes sociais são principalmente um meio de cultivar os relacionamentos pessoais e de se divertir tanto para adolescentes de escolas públicas como privadas. Apenas o motivo de uso fazer download de músicas e filmes apresentou diferença significativa entre os grupos. Essa diferença pode ser explicada pelo tipo de conexão a que os adolescentes de escola pública têm acesso que, provavelmente, é mais lento. 
Os jovens também, algumas vezes, buscam as redes sociais para "poder abstrair das frustrações e correrias do dia-a-dia", "se sentir melhor quando estão tristes ou abatidos" e "evitar a solidão". Contudo, apesar de alguns estudos (Bargh, Mckenna \& Fitzsimons, 2002; Kraut, Kiesler, Boneva, Cummings, Helgeson \& Crawford, 2002) sugerirem que a internet, através dos seus diferentes recursos comunicacionais, está promovendo modificações importantes na forma das pessoas se relacionarem, especialmente no que tange à revelação de aspectos pessoais e íntimos do si mesmo, isso não foi identificado nesse estudo. As redes sociais podem ser eventualmente utilizadas para lidar com problemas com os quais se deparam. No entanto, quando os jovens utilizam as redes sociais com essa finalidade, parece que o fazem conversando com pessoas conhecidas, como sugere o estudo de Dias (2003).

\section{CONSIDERAÇÕES FINAIS}

Os resultados indicaram que o nível socioeconômico influenciou na frequência de acesso às redes sociais, visto que alunos com baixa renda familiar podem ter maior dificuldade de aquisição de recursos materiais (computador) e de acesso rápido a internet e redes sociais. Entretanto, apesar de não terem o computador em casa, os alunos de escolas públicas não deixaram de acessar as redes sociais, procurando se inserir no espaço cibernético através de diferentes alternativas de uso. As escolas, bibliotecas, shoppings e lan houses (locais comerciais destinados às pessoas que querem utilizar computador) oferecem acesso à internet, muitas vezes, de forma gratuita ou com um mínimo custo. Isto amplia a oferta e facilita o acesso a esse recurso.

A renda familiar é uma importante variável a ser levada em consideração para entender o fenômeno da utilização das redes sociais pelos adolescentes. Entretanto parece não existir uma diferença qualitativa no uso da internet entre os alunos que apresentavam diferenças em relação à renda familiar e frequentavam diferentes tipos de escola (públicas e privadas). Apesar da menor frequência de acesso relatada pelos alunos de escolas públicas quando comparado aos alunos de escolas privadas, os adolescentes pareceram apresentar os mesmos motivos, acessar os mesmos sites e realizar as mesmas atividades na rede, o que sugere uma democratização desse recurso. Na opinião dos adolescentes as redes sociais são importantes meios de comunicação e diversão.

Mais pesquisas sobre como as redes sociais podem estar modificando as formas dos adolescentes se relacionarem com os outros, com o mundo e talvez até mesmo com eles próprios devem ser realizadas. Este estudo identificou que os jovens utilizavam as redes sociais para eventualmente lidar com frustrações e problemas do dia a dia. Porém, não foi investigado como isso é realizado pelos jovens. Com quem esses adolescentes desabafam ou procuram ajuda nessas redes sociais? Além disso, poderia ser investigado: como os jovens vivenciam os feedbacks sobre suas identidades oferecidas nas interações via rede social? Ou então: como as interações estabelecidas no contexto das redes sociais são afetadas pela realização de atividades múltiplas e paralelas, como troca de mensagens simultâneas com diferentes interlocutores? Ainda, a interação "rede social e adolescência" é um fenômeno incessante, uma vez que a dinamicidade de criações tecnológicas e virtuais é alta e age bidirecionalmente no desenvolvimento psicológico e social dos adolescentes. Isso demanda estudos constantes que caminhem lado a lado com a evolução das relações virtuais. Essas são algumas questões que podem ser exploradas em futuros estudos.

\section{REFERÊNCIAS}

Allen, J. P., Evans, M. A., Hare, A. L., \& Mikami, A. Y. (2010) Adolescent peer relationships and behavior problems predict young adult's communication on social Networking websites. Development Psychology, 46,46-56.

Amorim, W. A. (2009). O uso do website orkut como uma fonte de informação acadêmica. Manuscrito não publicado. Universidade Federal do Rio de Janeiro, Rio de Janeiro.

Bargh, J. A., McKenna, K. Y. A., \& Fitzsimons, G. M. (2002). Can you see real me? Activation and expression of the "True Self" on the internet. Journal of Social Issues, 58, 33-48.

Barros, C. C., \& Neto, J. L. F. (2010). Adolescência e MSN: O arranjo tecnológico da subjetividade. Pesquisas e Práticas Psicossociais, 5, 30-38.

Blais, J. J., Craig, W. M., Pepler, D., \& Connolly, J. (2008). Adolescents online: The importance of internet activity choices to salient relationships. Journal Youth Adolescence, 37, 522-536.

Barmage, D. F. A. (2010). A constituição do sujeito adolescente e as apropriações da Internet: uma análise histórico-cultural. (Dissertação de Mestrado). Universidade Federal do Mato Grosso do Sul. Campo Grande.

Bauman, Z. (2004). Amor líquido: Sobre a fragilidade dos laços humanos. Rio de Janeiro, Zahar. 
Delaunay, G. J. (2008). Novas tecnologias, novas competências. Educar em revista, Curitiba, 31, 277-293.

Clarke-Pearson, K., \& O’keeffe, G. S. (2011). Clinical Report The Impact os Social Media on Children, Adolescents and Families. Pediatrics, 127, 800-804.

Datafolha (2013). Jornal e educação. Retirado de http://www.anj.org.br/jornaleeducacao/noticias/pesquisa-dodatafolha-aponta-as-principais-fontes-de-informacao-dosjovens/

Dias, A. C. G. (2003). A revelação de si na internet, um estudo com adolescentes. (Tese de Doutorado). Universidade de São Paulo, São Paulo.

Eisenstein, E., \& Estefenon, S. (2006). Computador: Ponte social ou abuso virtual? Adolescência e Saúde, 3 .

Erikson, E. H. (1972). Identidade, juventude e crise. Rio de Janeiro: Guanabara, 1987.

Freitas, M. T. A. (2005). Sites construídos por adolescentes: Novos espaços de leitura/ escrita e subjetivação. Cadernos CEDES, 25, 87-101.

Governo Federal (2010). Relatório de pesquisa quantitativa. Hábitos de informação e formação de opinião da população brasileira. Retirado de http://www.secom.gov.br/pesquisas/ 2010-12-habitos-ii/2010-12-habitos-de-informacao-eformacao-de-opiniao-da-populacao-brasileira-ii.pdf

Griffits, M. D. (2000). Excessive Internet use: Implications for sexual behavior. Cyberpsychology and Behavior, 3, 537-552.

Griffits, M. D. (1997). Children and internet. Media Education Journal, 21, 31-33.

IBGE: Instituto Brasileiro de Geografia e Estatística (2009). Retirado de http://www.ibge.gov.br/home/presidencia/noticias/ noticia_visualiza.php?id_noticia $=1708$.

Kraut, R., Kiesler, S., Boneva, B., Cummings, J., Helgeson, V., \& Crawford, A. (2002). Internet paradox revisited. Journal of Social Issues, 58, 49-74.

Kraut, R., Patterson, M., Lundmark, V., Kiesler, S., Mukopadhyay, T., \& Scherlis, W. (1998). Internet paradox: A social technology that reduces social involvement and psychological Well-Being? American Psychologist, 53, 1017-1031.

Levy, P. (1999). Cibercultura. São Paulo, edição 34, 2010.

Leon, D. T. \& Rotunda, R. J. (2000). Contrasting case studies of frequent internet use: Is it pathological or adaptative? Journal of College Student Psychoterapy, 14, 9-18.

Mello, H. D. (1997). Representação e uso da internet por adolescentes de Florianópolis. (Dissertação de Mestrado). Universidade Federal de Santa Catarina. Florianópolis.

Nicolaci, A. M. (2005). Sociabilidade virtual: Separando o joio do trigo. Psicologia e Sociedade, 17, 50-52.

Nicolaci, A. M. (1998). Na malha da rede: Os impactos íntimos da internet. Rio de Janeiro: Campus.
Nobrega, L. P. (2010). A construção das identidades nas redes sociais. Fragmentos de Cultura, 20, 95-102.

Ponte, C. (2010). Jovens e internet: discutindo divisões digitais. Em M. Barbosa, \& O.J. Morais. (Orgs). Comunicação, cultura e juventude (47- 71). São Paulo: Intercom.

Prilla, J. P. V., Muza, M. L. N., \& Campos-Antoniassi, P. I. (2011). Letramento on-line: As redes sociais conectadas ao processo de ensino e aprendizagem e a democratização do conhecimento na era da cibercultura. Trabalho não publicado. Universidade de Sorocaba, São Paulo.

Pujol, C. C.; Schimidt, A.; Skolovsky, A.; Karam, R. G., \& Spritzer, D. T. (2009). Dependência de internet: Perspectivas em terapia cognitivo-comportamental; Revista Brasileira de Psiquiatria, 31, 185-186.

Ramos, D. K., \& Silva, A. S. (2011). Comunicação, diversão e aprendizagem: Um estudo exploratório sobre o uso das tecnologias pelos adolescentes. Poiésis, 4, 405-421.

Recuero, R. (2009). Redes sociais na internet. Editora Meridional.

Regan, P., \& Sevees, V. (2010). Kids R Us: Online social networking and the potencial for empowerment. Surveillance \& Society, 8, 151-165.

Richardson, R. J. (1989). Pesquisa social: Métodos e técnicas. São Paulo: Atlas.

Spizzirri, R. C. P., Wagner, A., Mosmann, C. P., \& Armani, A. B. (2012). Adolescência conectada: Mapeando o uso da internet em jovens internautas. Psicologia Argumento, 30, 327-335.

Suzuki, F. T. I., Matias, M. V., Silva, M. T. A., \& Oliveira, M. P. M. T. (2009). O uso de videogames, jogos de computador e internet por uma amostra de estudantes universitários da Universidade de São Paulo. Jornal Brasileiro de Psiquiatria, 58, 162168.

Turkle, S. (1998). A vida no ecrã: A identidade na era da Internet. Lisboa: Relógio d'agua.

Valkenburg, P. M., \& Peter, J. (2007). Preadolescents and adolescents online communication and their closeness to friends. Development Psychology, 43, 267-277.

Young, K. (2007). Cognitive behavior therapy with Internet addicts: Treatment outcomes and implications; Cyberpsychology \& Behavior, 10, 671-679.

Wolak, J., Mitchell, K. J., \& Finkelhor, D. (2003). Escaping or connecting? Characteristics of youth who form close online relationships. Journal of Adolescence, 26, 105-119.

World Health Organization. Adolescent health \& development. Retirado de http://www.who.int/topics/adolescent_health/en/ >.

Recebido em 09/07/2012

Primeira Decisão Editorial em 29/04/2013

Aceito em 05/05/2013 